\title{
Coenzym Q10 ohne neuroprotektiven Effekt
}

Fragestellung: Ziel der vorliegenden Studie war es, zu untersuchen, ob die Hochdosisbehandlung mit Coenzym Q10 die Krankheitsprogression bei Morbus Huntington verlangsamen kann.

Hintergrund: Eine Vielzahl experimenteller Daten deuten auf einen gestörten Energiestoffwechsel als ein Element der Pathophysiologie bei Morbus Huntington hin. Folglich müssten Substanzen, die die mitochondriale Funktion verbessern und den oxidativen Stress reduzieren, Kandidaten für eine neuroprotektive Studie bei der Erkrankung darstellen. Die Annahme wird durch positive Effekte solcher Substanzen im Mausmodell unterstützt. Coenzym Q10 zeigte positive Effekte in experimentellen Modellen der amyotrophen Lateralsklerose, der ParkinsonErkrankung und auch in einigen Huntington-Modellen. Neben anderen Effekten spielt Coenzym Q10 eine zentrale Rolle bei der oxidativen Phosphorylierung, scheint membranstabilisierend und antioxidativ zu wirken. Darüber hinaus zeigte sich in einer früheren, kleineren, vierarmigen Studie (CARE-HD, $\mathrm{n}=347$ ) mit Coenzym Q10 (600 mg/

McGarry A, McDermott M, Kieburtz K et al. A randomized double-blind, placebo-controlled trial of coenzyme Q10 in Huntington disease. Neurology 2017; 88: $152-9$
Tag) und Remazemid ein Trend zu einer stabilisierenden Wirkung in funktionellen Skalen.

Patienten und Methodik: 609 Teilnehmer wurden in diese randomisierte, doppelblinde, placebokontrollierte Multicenterstudie eingeschlossen. In der Verumgruppe wurden die Teilnehmer mit 2.400 mg Coenzym Q10 täglich behandelt. Die Studiendauer betrug 60 Monate. Einschlusskriterien waren unter anderem eine molekulargenetisch gesicherte Diagnose und eine stabile Psychopharmakamedikation. Primärer Outcome war ein Unterschied in der funktionellen Skala „Total Functional Capacity“.

Ergebnisse: In einer Zwischenanalyse zeigte sich, dass nur eine Wahrscheinlichkeit von weniger als $5 \%$ besteht, dass die Studie am Ende ein positives Ergebnis aufzeigen würde. Zur Zeit der Studienbeendigung hatten $91 \%$ der Patienten ein Jahr der Follow-up-Zeit, $83 \%$ zwei Jahre, $69 \%$ drei Jahre, $52 \%$ vier Jahre und $34 \%$ fünf Jahre Studiendauer abgeschlossen. Es zeigten sich keine statistisch signifikanten Unterschiede zwischen den Behandlungsgruppen für die primären oder sekundären Outcomes, mit Ausnahme des Ergebnisses im Word-Lese-Test des Stroop-Tests, das als Einzelbefund als „nicht echt“ bewertet wurde. Zwar gab es mehr Tote in der Coenzym-Q10-Gruppe im Vergleich zur Placebogruppe, dieser Unterschied war jedoch nicht statistisch signifikant. Coenzym Q10 war generell sicher und gut verträglich.

Schlussfolgerung: Die Daten rechtfertigen nicht den Einsatz von Coenzym Q10 zur Behandlung der funktionellen Verschlechterung bei Morbus Huntington.

\section{- Kommentar von Carsten Saft, Bochum}

\section{Dauerhafte Coenzym-Q10-Einnahme scheint leider nicht sinnvoll}

Eine mitochondriale Dysfunktion wurde in mehreren Studien als wesentlicher Aspekt in der Pathophysiologie der Huntington-Erkrankung belegt. Lange Zeit wurden daher unter anderem eine Vitaminhochdosistherapie, Coenzym Q10 und Kreatin als potenziell schützende Substanzen empfohlen. Dies wurde durch teilweise ermutigende Befunde aus jedoch nur kleinen Studien unterstützt, die meist zu wenig Power und eine nur kurze Studiendauer hatten [1]. In der hier vorgestellten großen Studie konnte kein Einfluss auf die Krankheitsprogression belegt werden. Als mögliche Ursachen für den negativen Studienausgang werden eine nicht optimale Formulierung zur Resorption von Coenzym Q10, zu insensitive Studien-Readouts oder auch ein zu später Therapiebeginn diskutiert. So zeigte eine Phase-2-Studie im prämanifesten Stadium der Erkrankung mit Kreatin über 18 Monate Studiendauer einen möglicherweise positiven Effekt mit verminderter Hirnatrophie [2].

Auch Kreatin, ein anderes potenziell wirksames Präparat, wurde in diesem Zusammenhang in einer großen Studie mit 551 Huntington-Patienten in Dosen von bis zu $40 \mathrm{~g}$ täglich über drei Jahre getestet [3]. Auch diese Studie wurde wegen „Aussichtslosigkeit" nach einer Zwischenanalyse abgebrochen. Zu- sammenfassend erscheint damit die Einnahme von Coenzym Q10 oder Kreatin unter der Vorstellung eines neuroprotektiven Effekts nicht sinnvoll. Derzeit rekrutieren mehrere Studien, auch mit potenziell protektivem Ansatz, sodass die Vorstellung von Huntington-Patienten in einem Zentrum empfohlen wird.
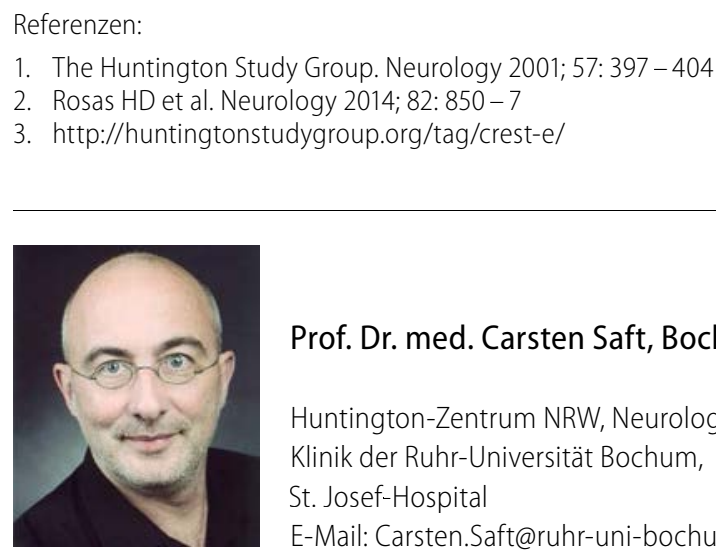

Prof. Dr. med. Carsten Saft, Bochum

Huntington-Zentrum NRW, Neurologische Klinik der Ruhr-Universität Bochum, St. Joset-Hospital E-Mail: Carsten.Saft@ruhr-uni-bochum.de 